- Article type: Paper

- $\quad$ Revised Date: October 2, 2017

- Words: 5,218

- Figures: 10

- Tables: 7

\title{
Use of a MEMS Accelerometer to Measure Orientation in a Geotechnical Centrifuge
}

Author 1

- Ryan D. Beemer, PhD

- ORCID: 0000-0002-9101-5325

- Centre for Offshore Foundation System, University of Western Australia, Crawley, Australia

- $\quad$ Work phone: +61 864882782

- Mobile phone: +61 0456411365

- Email: ryan.beemer@uwa.edu.au

Author 2

- Giovanna Biscontin, PhD

- ORCID: 0000-0002-4662-5650

- Department of Engineering, University of Cambridge, Cambridge, United Kingdom

Author 3

- Madhuri Murali, PhD

- ORCID: 0000-0002-1055-5072

- $\quad$ Project Engineer, Fugro Marine geo-Services, Inc., Houston, Texas

Author 4 
- Charles P. Aubeny, PhD

- ORCID: 0000-0002-4032-6895

- Zachry Department of Civil Engineering, Texas A\&M University, College Station, United States

\begin{abstract}
(181 words)
Microelectromechanical systems (MEMS) accelerometers are becoming more prevalent in geotechnical engineering and geotechnical centrifuge modelling. In centrifuge experiments these sensors have shown great promise, but still exhibit limitations. This paper proposes a new methodology for the use of single-axis, low-g, high accuracy MEMS accelerometers to measure orientation of on object on the vertical rotational plane of centrifugal acceleration and Earth's gravity in a geotechnical centrifuge. The method specifically compensates for measured cross-axis acceleration by a MEMS accelerometer when in a high-g environment. This is done by determining the apparent internal misalignment of the MEMS sensing unit, relative to its packaging, from a high-g cross-axis calibration. The misalignment can then be used to correct the measured orientation of sensor relative to a centrifuge gravity vector. When compared to simplified approaches measurements of absolute orientation are improved by $0.98^{\circ}$ and the standard deviation of measurements between multiple sensors is reduced by $0.73^{\circ}$. Overall, this new methodology significantly improves the accuracy of orientation measurements by a MEMS accelerometers in the geotechnical centrifuge, opening the door to use these inexpensive sensors in more experiments.

Keywords: Centrifuge modelling, Laboratory equipment, Monitoring
\end{abstract}

\title{
List of Notation
}

Y centrifuge axial coordinate

$r \quad$ centrifuge radial coordinate perpendicular to the centrifuge axis, $\mathrm{Y}$

$\omega \quad$ angular velocity of the centrifuge

$x \quad$ local horizontal coordinate of model

$y \quad$ local width coordinate of model

Z local vertical coordinate of model

Xsensor sensor X-coordinate 
ysensor sensor X-coordinate

$\mathrm{Z}_{\text {sensor }}$ sensor z-coordinate

$\mathrm{X}_{\mathrm{M}} \quad$ Apparent $\mathrm{X}$-coordinate of sensor due to misalignment

$R \quad$ vertical rotational inertial 2D reference frame defined by the centrifuge axis, $\mathrm{Y}$, and centrifuge radial axis, $r$

$g$ magnitude of centrifuge gravity vector, $\tilde{g}$, in the vertical rotational plane

$g_{c} \quad$ magnitude of centrifugal acceleration vector, $\tilde{g}_{c}$

$g_{e} \quad$ magnitude of Earth’s gravity vector, $\tilde{g}_{e}$

$\alpha \quad$ angle between a centrifuge gravity vector, $\tilde{g}$, and the centrifuge radial coordinate, $r$

$\beta \quad$ angle between a centrifuge gravity vector, $\tilde{g}$, and the local vertical coordinate, $z$

$\xi \quad$ angle between the local vertical coordinate axis, $z$, and the centrifuge radial coordinate, $r$

$V_{n} \quad$ measured voltage by a MEMS accelerometer due to an acceleration applied in its measurement direction

$V_{0} \quad$ measured zero-g voltage by a MEMS accelerometer when no acceleration is applied

$V_{\alpha} \quad$ measured zero-g voltage by a MEMS accelerometer with apparent internal misalignment and a cross-axis acceleration of $1 \mathrm{~g}$ is applied

$V_{x} \quad$ is the measured voltage from an applied cross-axis acceleration, $a_{x}$

$C_{F} \quad$ calibration factor relating applied acceleration to measured voltage (V/g)

$C_{F \alpha} \quad$ calibration factor relating applied acceleration to measured voltage (V/g) with internal misalignment and a zero-g voltage of $V_{\alpha}$

$C_{x} \quad$ cross-axis acceleration correlation factor

$\theta_{n} \quad$ angle of MEMS accelerometer $\mathrm{z}$-coordinate, $\mathrm{z}_{\text {sensor, }}$ to the centrifuge acceleration vector $\tilde{g}$

$\theta_{t} \quad$ angle of MEMS accelerometer $\mathrm{z}$-coordinate, $\mathrm{z}_{\text {sensor, }}$, to the model $\mathrm{z}$-coordinate, $\mathrm{z}$

$\theta_{\alpha} \quad$ the apparent internal angular misalignment of the MEMS accelerometer in the x-z plane

$a_{n} \quad$ an acceleration applied in-line with the sensors measurement directions

$a_{\text {meas }}$ acceleration measured by the MEMS accelerometer

$a_{\text {cross }}$ component of acceleration perpendicular to the MEMS accelerometer measurement direction, $\mathrm{z}_{\text {sensor, }}$ measure by the sensor 
$a_{\text {temp }}$ acceleration measured by the MEMS accelerometer due to temperature change of the sensor $a_{x} \quad$ magnitude of an applied cross-axis acceleration in $\mathrm{z}_{\text {sensor }}$

Vertical rotational plane A vertical plane defined by centrifuge axis, Y, and centrifuge radial coordinate, $r$ 


\section{Introduction}

2 Microelectromechanical systems (MEMS) accelerometers have become a ubiquitous part of

3 everyday life, being found in mobile phones, tablets and cars. Their prevalence in part is due to the mass production silicon fabrication techniques used to manufacture them (Spangler and Kemp 1996), which allows for low relative costs. Aside from their cost, MEMS accelerometers are an

6 attractive option for geotechnical engineers because to their ability to measure persistent

7 acceleration. Unlike piezoelectric accelerometers, MEMS can measure a vector of constant 8 acceleration and their orientation relative to this vector.

9 The adaptation of MEMS into civil engineering has been advocated since at least 2000 (Oppenheim et al. 2000). In geotechnical engineering, specifically, MEMS accelerometers have served two main

11 purposes: dynamic measurements of sensor motion and quasi-static measurements of sensor 12 orientation relative to gravity. MEMS accelerometers have been used both in the field and the laboratory by geotechnical engineers. Examples include: measuring wave propagation with custom packaged MEMS accelerometer circuits (Hoffman et al. 2006; Bhattacharya et al. 2012), measuring soil mass deformation using the shape-acceleration array (Bennett et al. 2009), measuring acceleration in liquefaction field tests (Saftner et al. 2008), measuring penetrometers deceleration for characterizing offshore sediments (Stark et al. 2009), and monitoring the installation of dynamically embedded plate anchors (Blake and O’Loughlin 2015).

An area of geotechnical testing which has recently seen growth in the use of MEMS accelerometers is centrifuge scale modelling. Results from this paper were used by Beemer (2016) to measure caisson cycling at rotational amplitudes of less than 0.5 degrees, Fig. 1 . Other examples include: evaluation of MEMS accelerometers in dynamic centrifuge testing (Stringer et al. 2010), seismic evaluation of pile reinforced slopes (Al-Defae and Knappett 2014), measuring model radial distance from the centrifuge axis and dead reckoning of a dynamically penetrated anchor in-line with centrifuge gravity (O’Loughlin et al. 2014), measurements of monopile rotation using high-g accelerometers (Lau 2015), and large angle anchor orientation in sand (Chow et al. 2015). 
Though these initial cases have been quite successful, there is still room for improvement. Stringer et al. (2010) noted that spurious accelerations were measured during centrifuge spin up and residual velocities, after integration of acceleration, were also measured at completion of the experiment, when the sensors were still. The accuracy of orientation measurements with MEMS accelerometers has also been relatively low. Chow et al. (2015) reported orientation with errors of $\pm 1^{\circ}-2 \cdot 5^{\circ}$. While Lau (2015) found it necessary to amplify the output of a 35 g MEMS accelerometer by a gain of 10 to collect useable data, and even with this additional circuity there were cases where their accuracy was too low to be of use. If the angular accuracy of the MEMS accelerometers in high-g could be improved when used in the centrifuge a number of interesting and difficult problems could be investigated, such as: measuring rotation of monopile for offshore wind turbines where serviceability tilts are limited to $0.5^{\circ}$ (DNV 2007) and lateral spreading of shallow slopes. In the past, slopes with angles as low as $0.6^{\circ}$ (Taboada-Urtuzuástegui and Dobry 1998) and $3^{\circ}$ (Stringer et al. 2010) have been studied in the centrifuge.

To date, measurements of orientation in the centrifuge (Lau 2015; Chow et al. 2015; Allmond et al. 2014) have utilized a simple sinusoidal relationship to relate measured acceleration to orientation relative to centrifuge gravity. This process was outlined by Allmond et al. (2014) who showed the method resulted in good correlation to angular measurements from linear displacement transducers, but little discussion of initial or absolute orientation of the sensor to centrifuge gravity is provided. Their method also specifically excludes measured cross-axis acceleration, which was later suggested to be significant at accelerations as low as $10 \mathrm{~g}$ (Beemer et al. 2015). Additionally, measured cross-axis accelerations could explain the extraneous accelerations measured during spin up by Stringer et al. (2010). This paper expands on earlier quasi-static orientation theories by compensating for measured cross-axis accelerations created by the apparent internal misalignment of the MEMS sensing unit within the housing.

It is also worth noting that measured cross-axis accelerations are incorporated into accelerographs measurements of earthquake motions (Wong and Trifunac 1977). Traditionally, accelerographs rely 
on three single degree of freedom pendulums to measure acceleration. In this simple macromechanical design cross-axis effects can be broken into two components: cross-axis sensitivity and internal misalignment. Cross-axis sensitivity is attributed to acceleration applied cross-axis to the pendulum's designated degree of freedom when it under goes a pseudo-static rotation see (Wong and Trifunac 1977). Internal misalignment of the pendulum's measurement axis with respect to the accelerograph’s local coordinates will also result in a measured cross-axis acceleration. Complete solutions based on pendulum physics and coordinate rotation relative to Earth’s gravity are available to calibrate for both cross-axis effects and internal misalignment; however, these are not readily applicable to MEMS accelerometers.

The main reason accelerograph methods are not applicable to MEMS accelerometers is that they do not rely on pendulums to measure acceleration. Their micromechanical structures are actually quite varied and their exact design is not typically provided to the user. Many systems are based on spring mass systems, with varying means of converting proof mass deflection to an electrical signal (Shaeffer 2013). There are even designs where a proof mass is not even needed; heat convection MEMS accelerometers rely on temperature gradients within a heated micro-chambers to measure acceleration (Leung et al. 1997; MEMSIC 2007). A second reason accelerograph methods are not applicable, is that MEMS accelerometers zero-g voltage cannot be separated from a voltage measured when a cross-axis acceleration of $1 \mathrm{~g}$ is applied, under typical laboratory conditions. The method presented in this paper overcomes these issues by assuming any cross-axis sensitivity of the MEMS is due solely to an apparent internal misalignment and through the performance of a high-g cross-axis calibration.

This paper examines the use of MEMS accelerometers to measure orientation within a geotechnical centrifuge and presents a methodology for measuring sensor orientation relative to centrifuge gravity to a high accuracy. This investigation is supported by results from high-g cross-axis experiments on single-axis low-g accelerometers. It was found that measured cross-axis acceleration due to apparent internal misalignment of the sensor has a significant impact on 
79 measurements of absolute angular orientation relative to centrifuge gravity.

80

\section{Background}

\subsection{The Centrifuge Gravity Field}

In this paper centrifuge gravity is treated as 2-dimensional on the vertical rotational inertial acceleration, $g_{c}$, and Earth's gravity, $g_{e}$, Equations $1-2$. Any out-of-plane accelerations are considered beyond the scope of this paper.

$g_{c}=\omega^{2} r$

1.

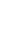

$g=g_{c} \cdot \hat{\imath}+g_{e} \cdot \hat{\jmath}$

\section{2.}

where: $g_{c}$ is centrifugal acceleration, $\omega$ is rotational velocity, $r$ is radial coordinate from the centrifuge axis, $g$ is centrifuge gravity, and $g_{e}$ is Earth's gravity about the basket hinge, such as from cabling and hydraulic hosing or changes to its centre-ofreference frame of the centrifuge axis and the radial coordinate and is the resultant of centrifugal

Additionally, this paper incorporates gravity field rotation due to tilt of a free-swinging centrifuge basket as presented in Beemer et al. (2016). That is, rotation of the basket due to applied moments gravity, will result in rotation of the model's coordinates, $\xi$, relative the radial coordinate, $r$. This will result in any centrifuge gravity vector, $\tilde{g}$, being at an angle $\beta$ to the model local coordinates $(x, z)$ as shown in Fig. 2. In the figure, $\alpha$ is the angle of centrifuge gravity to the centrifuge radial 
102

103

104

105

106

107

108

109

110

111

112

113

114

115

116

117

coordinate, $r$, and $R$ is the rotational reference frame.

\subsection{MEMS Accelerometers}

MEMS accelerometers convert a measured acceleration to electrical output. Unlike piezo-electric sensors, an input voltage must be applied for the sensor to work. Under a single-ended configuration they will output a constant signal at zero-g, known as the zero-g voltage, $V_{0}$. An acceleration measurement is then taken as:

$a_{n}=\left(V_{n}-V_{0}\right) \cdot C_{F}$

3.

where: $a_{n}$ is an acceleration applied in-line with the sensor's measurement directions, $V_{n}$ is the voltage measured due to an acceleration in-line with the sensor and $C_{F}$ is the calibration factor due to an acceleration applied in-line with the sensor.

The calibration factor is the linear relationship between measured voltage and applied acceleration and can be determined in two ways. The first is to apply quantities of known acceleration directly in-line with sensor's measurement direction and record the output voltage, Eq. 4. This could be done by placing the sensor at a known radius in a geotechnical centrifuge spinning at a precise angular velocity. This method allows for a MEMS accelerometer to be calibrated over its entire sensing range and ensures no cross-axis acceleration is measured. When calibrating low-g accelerometers with centrifugal acceleration, the angle of the vector relative to the sensor must be considered. In a drum centrifuge (or beam centrifuge with a fixed basket) the angle of centrifuge gravity, $\alpha$, to the accelerometer's measurement direction will be $45^{\circ}$ at $1 \mathrm{~g}, 11.3^{\circ}$ at $5 \mathrm{~g}$ and $5.7^{\circ}$ at $10 \mathrm{~g}$. Care must also be taken in beam centrifuges with free-swinging baskets. A basket is 
$129 \quad C_{F}=\frac{\Delta a_{n}}{\Delta V_{n}}$ 4. where: $C_{F}$ is the calibration factor

The second and more frequently used method is to rotate the accelerometer in Earth's gravity such that the applied acceleration ranges between $-1 \mathrm{~g}$ and $1 \mathrm{~g}$. A multi-point calibration can be done by the fabrication of an angular calibrator such as the 3D printed one shown in Fig. 3, used in the laboratories at the University of Western Australia and Texas A\&M University. It allows for a seven calibration points from $0^{\circ}$ to $90^{\circ}$, at $15^{\circ}$ increments. The disadvantage of this approach is that the magnitude of acceleration applied to a sensor is limited to $\pm 1 \mathrm{~g}$, which is just a fraction of the range of a $5 \mathrm{~g}$ or $10 \mathrm{~g}$ MEMS accelerometer. This method is typically preferred because it is cheaper and more time effective to calibrate the accelerometers outside the centrifuge, especially given it is best practice to re-calibrate sensors on a regular basis.

\section{Accelerometer Orientation Theory in the Centrifuge}

A quasi-static assumption is used in this derivation. As such, kinematic accelerations from relative displacement or rotation of the accelerometer are not considered and are outside the scope of this paper. This includes Coriolis acceleration, which is dependent on sensor velocity along the centrifuge radial coordinate, $r$. For more on Coriolis accelerations in the centrifuge see Madabhushi 
149 (2015), Randolph et al. (1991), and Schofield (1980).

150

3.1 Sensor Measurements and Geometry

Measurements of orientation by a single-axis MEMS accelerometer are made relative to an acceleration vector, in this case centrifuge gravity. Ideally, when the sensor is perpendicular to a centrifuge gravity vector it should read zero and when it is in-line with a centrifuge gravity vector it should read its magnitude. However, in a high-g environment this is not the case. Actual measurements from a MEMS accelerometer are affected by a number of factors, as shown in Fig. 4: applied centrifuge gravity, $\tilde{g}$, measured acceleration due to sensor change in temperature, $a_{\text {temp }}$, and any measured accelerations due to cross-axis sensitivity, $a_{\text {cross. }}$ The measured cross-axis acceleration results from the sensor's tendency to measure a portion of an acceleration applied perpendicular to its measurement direction, $\mathrm{z}_{\text {sensor }}$ in Fig. 4. Combining these, the measured acceleration from a single-axis MEMS accelerometer will be:

where: $a_{\text {meas }}$ is the acceleration measured by the MEMS accelerometer, $a_{\text {cross }}$ is the measured cross axis acceleration and $a_{\text {temp }}$ is the acceleration measured due to temperature change of the sensor

If it is then assumed that all measured cross-axis acceleration can be modelled as an internal misalignment of the MEMS sensing unit within the package, Fig. 5, Equation 5 can be presented dependent on the sensor's angle to centrifuge gravity, Equation 6. This assumption appears valid 170 given the linearity of measured cross-axis acceleration with applied centrifuge gravity presented by 171 Beemer et al. (2015). 


$$
a_{\text {meas }}=g \cdot \sin \left(\theta_{n}+\theta_{\alpha}\right)+a_{\text {temp }}
$$

6.

where: $\theta_{\alpha}$ is the apparent misalignment and $\theta_{n}$ is the angular orientation of the sensor relative to centrifuge gravity

This is similar to the solution provided by Allmond et al. (2014); however, temperature effects and measured cross-axis acceleration due to sensor internal misalignment are included. To determine a MEMS accelerometer's orientation relative to a centrifuge gravity vector, $\tilde{g}$, Equation 6 can be solved for $\theta_{n}$ :

$\theta_{n}=\arcsin \left(\frac{a_{m e a s}-a_{t e m p}}{g}\right)-\theta_{\alpha}$ 7.

The measured acceleration due to variation in sensor temperature is often insignificant (see Discussion); however, for completeness it is included in the final solution, Equation 7.

\subsection{Cross-Axis Sensitivity due to Internal Misalignment}

To determine the absolute orientation of an MEMS accelerometer in a high-g environment it is necessary to assess the effects of cross-axis sensitivity. It is assumed that all of the measured crossaxis acceleration is due to the apparent internal misalignment of the sensing unit within the package in the $\mathrm{x}-\mathrm{z}$ plane, about the $\mathrm{y}_{\text {sensor }}$ axis, Fig. 5. In actuality, cross-axis sensitivity can be the result of both intrinsic mechanical effects and internal misalignment. If the entirety of the reported cross-axis sensitivity for the $10 \mathrm{~g}$ accelerometers used in this paper, Table 1 , is assumed to be the result of 
196 misalignment then an apparent internal misalignment of $\pm 2.86^{\circ}$ is possible.

197 The component of cross-axis acceleration in the sensor's measurement direction for of an applied

198 cross-axis acceleration, $a_{x}$, in the $\mathrm{z}_{\text {sensor }}$ direction, Fig. 5, will be:

199

$200 \quad a_{\text {cross }}=\sin \left(\theta_{\alpha}\right) \cdot a_{x}$

2018.

202

203 where: $a_{x}$ is the magnitude of an applied cross-axis acceleration

204 Given an apparent internal misalignment, the expected measured misalignment of the sensor from

205 Equation 3 is:

206

$207 a_{\text {cross }}=\left(V_{x}-V_{0}\right) \cdot C_{F}$

2089.

209

210 where: $V_{x}$ is the measured voltage from an applied cross-axis acceleration, $a_{x}$

211 Setting Equation 8 equal to Equation 9 we can solve for the misalignment:

$213 \quad \theta_{\alpha}=\arcsin \left(\frac{V_{x}-V_{0}}{a_{x}} \cdot C_{F}\right)$

21410.

216 It can be seen that the first factor in the trigonometric function is the gradient of the measured cross- 
217 axis voltage to applied cross-axis acceleration. For the purpose of sensor calibration it is more

218 convenient and beneficial to define Equation 10 in terms of this quantity:

$220 \quad \theta_{\alpha}=\arcsin \left(C_{x} \cdot C_{F}\right)$

22111.

$223 \quad C_{x}=\frac{\Delta V_{x}}{\Delta a_{x}}$

22412.

226 where: $C_{x}$ is the cross-axis calibration factor

\subsection{Internal Misalignment and the MEMS 1-g Calibration Method}

229 Calibrating a MEMS accelerometer by rotating it in Earth’s gravity, Fig. 3, will incorporate the 230 apparent internal misalignment into the calibration variables. Therefore, it is necessary to assess the 231 effect misalignment on the zero-g voltage, $V_{0}$, and calibration factor, $C_{F}$, and how it can be 232 calculated when the 1-g calibration method is used.

233 It is assumed that the zero-g voltage is assessed by holding the sensor's measurement axes 234 perpendicular to Earth’s gravity. Given this, the zero-g voltage including misalignment is:

$236 \quad V_{\alpha}=V_{0}+\frac{\sin \left(\theta_{\alpha}\right) \cdot 1 \mathrm{~g}}{C_{F}}$ 
239 With a small angle assumption:

$241 \quad V_{\alpha}=V_{0}-\frac{\theta_{\alpha}}{C_{F}}$

244 where: $V_{\alpha}$ is the zero-g voltage with apparent internal misalignment and 1-g of applied cross-axis 245 acceleration

246 Depending on the sensitivity and noise of the individual sensor, it may be appropriate to assume the 247 second term in Equation 14 is negligible; however, this should be assessed on a case by case basis. 248 Next, it is necessary to assess the effect of misalignment on the calibration factor $C_{F}$, Equation 4 . If 249 the sensor is internally misaligned as in Fig. 5, the measured calibration factor with a misalignment $250 \theta_{\alpha}$ will be:

$C_{F \alpha}=\frac{1 g \cdot \sin \left(\theta_{2}+\theta_{\alpha}\right)-1 g \cdot \sin \left(\theta_{1}+\theta_{\alpha}\right)}{V_{2}-V_{1}}$

255 where: $C_{F \alpha}$ is the calibration factor with internal misalignment and a zero-g voltage of $V_{\alpha}, \theta_{i}$ are the 256 angles at which the accelerometers are calibrated, and $V_{i}$ are sensor output voltages at angles $\theta_{i}$ 257 Substituting in trigonometric identities and simplifying: 
$259 C_{F \alpha}=\frac{\left(\sin \left(\theta_{2}\right) \cos \left(\theta_{\alpha}\right)+\cos \left(\theta_{2}\right) \sin \left(\theta_{\alpha}\right)\right)-\left(\sin \left(\theta_{1}\right) \cos \left(\theta_{\alpha}\right)-\cos \left(\theta_{1}\right) \sin \left(\theta_{\alpha}\right)\right)}{V_{2}-V_{1}}$ 26016.

262 A small angle assumption can then be applied, assuming as well that $\cos \left(\theta_{\alpha}<3^{\circ}\right)=1$ :

263

264

$C_{F \alpha}=\frac{\sin \left(\theta_{2}\right)-\sin \left(\theta_{1}\right)+\cos \left(\theta_{2}\right) \theta_{\alpha}-\cos \left(\theta_{1}\right) \theta_{\alpha}}{V_{2}-V_{1}}$

Rearranging Equation 17 produces the following:

$$
C_{F \alpha}\left(\frac{V_{2}-V_{1}}{\sin \left(\theta_{2}\right)-\sin \left(\theta_{1}\right)}\right)=1+\theta_{\alpha}\left(\frac{\cos \theta_{2}-\cos \theta_{1}}{\sin \theta_{2}-\sin \theta_{2}}\right)
$$

18.

272 Upon inspection it can be seen that the second factor on the left-hand side is inverse of the

273 calibration factor, Equation 4 . Additionally, $\theta_{1}$ and $\theta_{2}$ can be set to $0^{\circ}$ and $90^{\circ}$, respectively,

274 encompassing the full 1-g calibration range. Substituting and simplifying:

$276 \quad C_{F \alpha}=C_{F}\left(1-\theta_{\alpha}\right)$

27719. 
279 This shows that the internal misalignment has an impact on the calibration factor. For an apparent 280 misalignment of $2^{\circ}$ the error could be up to $3.5 \%$.

281 With $C_{F \alpha}$ now known the misalignment, Equation 11, can be updated with Equation 19:

$\theta_{\alpha}=\arcsin \left(C_{x} \cdot \frac{C_{F \alpha}}{1-\theta_{\alpha}}\right)$

Applying a small angle assumption and rearranging:

291 Knowing that the misalignment, $\theta_{\alpha}$, will be less than 0.5 radians, Equation 21 can be solved with the 292 quadratic formula:

$\theta_{\alpha}=\frac{1-\sqrt{1-4 \cdot C_{x} \cdot C_{F \alpha}}}{2}$

296 To obtain the sensor misalignment, $\theta_{\alpha}$, the corrected calibration factor, $C_{F}$, and the zero-g voltage, $297 V_{0}$, all that is require is for a user to measure the cross-axis correlation factor, $C_{x}$, by measuring the 298 output voltage over a range of applied cross-axis accelerations.

299 It is also possible for the MEMS unit within the sensor package to be misaligned in the x-y plane, 
about the $\mathrm{z}_{\text {sensor }}$ axis, and in the $\mathrm{y}-\mathrm{z}$ plane, about the about the $\mathrm{x}_{\text {sensor }}$ axis. It can be shown the impact

301 of these internal misalignments are insignificant, for small angles, but these solutions are considered outside the scope of this paper.

\subsection{Orientation Relative to Basket Local Coordinates}

305 As noted previously the MEMS accelerometer orientation, $\theta_{n}$, is relative to the centrifuge gravity

306 vector at the location of the sensor. To determine the orientation relative to the local vertical coordinate, $z$, it is necessary to take into account rotation, $\beta$, of the model local coordinate $(x, z)$ to the local coordinate system $(x, z)$ as in Fig. 4 can then be defined as:

320 where: $\theta_{t}$ is the orientation of the sensor relative to the local $(\mathrm{x}, \mathrm{z})$ coordinate and $\beta$ is the angle

321 between the centrifuge gravity vector, $\tilde{g}$, and the local vertical coordinate, $z$ 


\section{Validation Testing Program}

The MEMS accelerometer selected to be the representative model is the MEMSIC CXL10GP1 single-axis accelerometer (MEMSIC n.d.) with a $\pm 10 \mathrm{~g}$ range, to further be referred to as $10 \mathrm{~g}$ Accelerometer; nine were used in the experiment. A single axis Silicon Design Model 2012 (Silicon Design Inc. 2013) with \pm 100 g range of was used to measure applied acceleration, to be referred to subsequently as $100 \mathrm{~g}$ Accelerometer. Technical specifications for the $10 \mathrm{~g}$ and $100 \mathrm{~g}$ accelerometers can be found in Table 1.

Experiments were conducted in the 150 g-ton, $2.7 \mathrm{~m}$ nominal radius, beam type centrifuge at Rensselaer Polytechnic Institute in Troy, NY (Elgamal et al. 1991). Three custom 3D printed ABS plastic Test Platforms were used to carry the nine $10 \mathrm{~g}$ Accelerometers while the $100 \mathrm{~g}$ accelerometer was mounted to a separate platform, Fig. 6. The initial calibration factors and zero-g voltage with apparent internal misalignment are provided in Table 2. The 10 g Accelerometer platforms each carried three $10 \mathrm{~g}$ Accelerometers: two parallel to the basket floor and one inclined at $4^{\circ}$. All of the platforms were secured to the floor of the metal centrifuge basket with small (adhesive backed) rare earth magnets. The platforms were centred in the basket such that their $\mathrm{x}-\mathrm{z}$ plane aligned with the plane of the centrifugal acceleration and Earth’s gravity $(r, \mathrm{Y})$, Fig. 7.

Three experiments were conducted. Each involved a single spin of the centrifuge where gravity, $\tilde{g}$, was stepped up in order to record the magnitude of acceleration measured by the $10 \mathrm{~g}$ Accelerometers. Accelerations were selected at regular intervals decreasing in step size at higher-g levels. Applied accelerations were monitored with the $100 \mathrm{~g}$ Accelerometer, Table 3. It was assumed that angle $\beta$ between the centrifuge gravity, $\tilde{g}$, at the sensors and the model local coordinates was sufficiently small as not to impact measurements. Applied accelerations were not incremented at whole numbers because the sensors were beyond the centrifuge nominal radius which the control software uses when setting the rotational velocity. Between the experiments the $10 \mathrm{~g}$ Accelerometers were rotated from the zero degree spots on the platform to the four degree spots as outlined in Table 4, platforms are as numbered in Fig. 6. 


\section{Results}

351 A cursory examination of the data collected from the sensors held at zero degrees in Experiment 352 Three provided some interesting results, Fig. 8. If the assumption that measured cross-axis acceleration were insignificant were true all of the sensors would have recorded zero voltage over the course of the experiment. However, it can be clearly seen this was not the case. Cross-axis acceleration up to $475 \mathrm{mV}$ was measured, in the case of M7, which is $12 \%$ of the $10 \mathrm{~g}$ Accelerometer output voltage range, Table 1. It can also be seen that magnitude of measured crossaxis acceleration is not the same for all sensors and can even be negative, as in the case of M8. This variation indicates that the measurements were not simply due to tilt of the centrifuge basket, $\xi$.

\subsection{Cross-Axis Correlation and Senor Internal Misalignment}

Initial calibration of internal misalignment showed consistent differences in measurements in

Experiment One relative to Experiments Two and Three, Table 5. This uniformity indicates that the angle of centrifuge gravity relative to the sensors vertical axis, $\beta$, was $0.22^{\circ}$ larger during Experiment One. This was due to the centrifuge basket being tilted at a different angle during that specific test. Any variation in $\beta$ will have the same result as an apparent internal misalignment, $\theta_{\alpha}$, and can be corrected for. It was assumed that the angle $\beta$ during Experiment Two and Three was closer to zero and Experiment One was correct for a $0.22^{\circ}$ angle.

A nearly linear relationship can be seen between measured cross-axis acceleration, $a_{\text {cross, }}$, and centrifuge gravity, $\tilde{g}$, Fig. 9, especially at higher accelerations. Linear curve regression fitting was carried out for data above 65g for all experiments, to determine the cross-axis calibration factors,

371 Table 6. These specific sensors were being calibrated for use in a 70 g experiment. It can be seen that the correlations show a high order of linearity, with M8 being the lowest with an $\mathrm{R}^{2}$ of 0.973 . 
provided in Table 6. The apparent misalignment lies within manufacture specification, Table 1, ranging from $-0.16^{\circ}$ to $1.61^{\circ}$ with a mean and standard deviations of $0.86^{\circ}$ and $0.62^{\circ}$, respectively.

\subsection{Model Validation}

As previously noted, three of the nine $10 \mathrm{~g}$ Accelerometers were held at a four degree angle during each experiment to test the hypothesis that measured cross-axis acceleration could be corrected for apparent misalignment. Fig. 10 presents the results, which are grouped by testing platform and tabulated in Table 7. Temperature effects were considered negligible and a temperature correction was not included (see discussion for more).

The results of the comparison clearly show that the cross-axis sensitivity is not negligible and contributes significantly to the magnitude of the measured angle. The average measurement of the $4^{\circ}$ shelves is $3.02^{\circ}$ when cross-axis sensitivity is neglected and $3.94^{\circ}$ when it is considered. This is a 23\% improvement in measurement precision, if the 3D printed platforms are indeed at an angle of $4^{\circ}$ (see Discussion). More significant, however, is the scatter in the uncorrected results when comparing sensors, as seen in Fig. 10. For measurements where cross-axis effects are ignored, the standard deviation in the measurement of the platform angle is $0.73^{\circ}$, on average, while it is only $0.02^{\circ}$ when a correction is made for misalignment. Though in absolute terms this error is not large, it is significant relative to the desired measurement quantity in serviceability limits.

\section{Discussion}

\subsection{Sensor Accuracy}

Accuracy of orientation measurements with MEMS accelerometers is dependent on the data acquisition system (DAQ), sensor accuracy, sensor orientation, and magnitude of centrifuge gravity, $\tilde{g}$. In general, any sensor will only be as accurate as the measurement capabilities of the DAQ 
sampling it; this has been specifically discussed for MEMS accelerometers by O’Loughlin et al.

399 (2014). Each model of MEMS accelerometer will have an intrinsic measurement accuracy

400 dependent on its output noise and offset. Sensor angular accuracy will be highly impacted by the

401 initial orientation of the accelerometer. If the sensors measurement direction is initially in-line with

402 centrifuge gravity a low accuracy, high range sensor will be required. However, if the sensor is

403 initially aligned perpendicular to gravity a high accuracy, low range accelerometer can be used.

404 Additionally, the sinusoidal functions relating centrifuge gravity to orientation are more variable

405 when rotating into an acceleration vector than away from it. That is, the sine of a small angle is 406 more variable than the cosine of a small angles. The accuracy of orientation measurements is also

407 highly dependent on the magnitude of centrifuge gravity, as seen in Equation 24. Measurements of 408 tilt from a MEMS accelerometer will increase in accuracy for increasing magnitudes of centrifuge 409 gravity; this in turn will decrease the accelerometer's angular range. For example, if the $10 \mathrm{~g}$ 410 Accelerometer accuracy is taken as three time the noise, Table 1, then its accuracy would be 411 approximately $0.12^{\circ}$ at $50 \mathrm{~g}$ and $0.06^{\circ}$ at $100 \mathrm{~g}$ while its range would be approximately $11.54^{\circ}$ at $41250 \mathrm{~g}$ and $5.74^{\circ}$ at $75 \mathrm{~g}$.

\subsection{Influence of Temperature on Sensor}

As seen in Table 1, environmental temperature can influence the reading of MEMS accelerometers. Though this effect should be considered on a case by case basis, in general it should be minimal. This is in part due to the fact that major beam centrifuges are ventilated to prevent excessive 418 temperatures (Elgamal et al. 1991; Ellis et al. 2006; Madabhushi 2015; Randolph et al. 1991; 419 Schofield 1969; Black et al. 2014). From the literature, a worst case temperature variation for a 420 centrifuge experiment appears to be taking a sensor from room temperature $\left(25^{\circ} \mathrm{C}\right)$ to a refrigerated 421 centrifuge model. Barrette et al. (1999) reduced a centrifuge model’s temperature to $-10^{\circ} \mathrm{C}$, or a 422 differential of $35^{\circ} \mathrm{C}$, from room temperature. Given the $10 \mathrm{~g}$ Accelerometer, Table 1, this would 423 result in an approximate apparent measured angle of about $0.34^{\circ}$ at $50 \mathrm{~g}$. In this case it could be 
reasonable to include the effect of temperature.

\subsection{Experimental Validation of Model}

427 Results from the validation show that the proposed model can be used to measure orientation in the

428 centrifuge environment and that the inclusion of cross-axis sensitivity significantly improves

429 measurements of orientation. Measured angle with the cross-axis correction of all the platforms

430 were close to the design angle of $4^{\circ}$ : Platform One was $3.81^{\circ}$, Platform Two was $4.03^{\circ}$, and Platform

431 Three was $3.96^{\circ}$, or a $0.09^{\circ}$ on average difference with most of the error in Platform One. This is a

432 significant improvement over the $0.98^{\circ}$ on average difference when cross-axis effects from apparent

433 misalignment are ignored. Even more significant is that the average standard deviation of the

434 measurement of these $4^{\circ}$ platforms across all spins/experiments is $0.02^{\circ}$, on average, with the cross-

435 axis correction and is found to be $0.73^{\circ}$, without cross-axis correction.

436 Given the low standard deviation in the platform measurements across all the experiments it appears 437 the 0.19 error in Platform One is due to the tolerances in the 3D printing process. The tolerance in 438 3D printing processes of the platforms was $\pm 0.127 \mathrm{~mm}$ (Stratasya 2015). Given this, the maximum 439 possible error between the two legs, $70 \mathrm{~mm}$ apart, holding the sensor at $4^{\circ}$ would be $0.254 \mathrm{~mm}$ and 440 the maximum angular error would be $0.21^{\circ}$ and this could account for all the error seen on Platform 441 One tests. Additional error could be introduced from the deformation of the platform under high-g 442 or by tolerance in the thickness of the rare earth magnets used to fix the platforms to the centrifuge 443 basket. It is recommended that calibration platforms are constructed to a higher precision when 444 working with these high accuracy sensors.

\section{Conclusions}

447 A number of conclusions can be drawn from the updated quasi-static orientation theory for single448 axis MEMS accelerometers, the determination of cross-axis correlation factors, and the validation 
experiment.

1. Single-axis MEMS accelerometers will measure significant magnitudes of cross-axis acceleration as a reaction to centrifuge gravity applied perpendicular to their measurement direction, Fig. 10. This can be attributed to an apparent misalignment of the sensing unit within the sensor package, Table 6 . In these experiments a maximum error of $1.69^{\circ}\left(0.98^{\circ}\right.$ on average) was seen when cross-axis acceleration from apparent internal misalignment was neglected, Table 7 . Additionally, a standard deviation of $0.73^{\circ}$ was seen in measurements when cross-axis acceleration was neglected, instead of the $0.02^{\circ}$ when included. Errors of this magnitude would be significant for experiments where serviceability limits are of concern or experiments on shallow slopes.

2. The apparent internal misalignment of a MEMS accelerometer can be measured with a highg cross-axis correlation, Equation 11-12 when the sensor is calibrated directly in-line with its measurement direction (this could be done in a centrifuge) and Equations 12 and 22 when the accelerometer is calibrated by rotating in Earth’s gravity, Fig. 3. The measured misalignment can then be used to correct the reading of absolute orientation from a MEMS accelerometer used in the high-g environment of a geotechnical centrifuge, Equation 24.

3. Low-g single-axis MEMS accelerometers can be used to make fine measurements of orientation in a high-g environment when rotated into centrifuge gravity. In this paper it was possible to measure the absolute orientation of a platform constructed at a $4^{\circ}$ angle to the basket floor to a standard deviation/accuracy of $0.02^{\circ}$ while centrifuge gravity was greater than $65 \mathrm{~g}$, Table 7. In fact it appears that they were sensitive enough to measure the tolerances in the $3 \mathrm{D}$ printing process used to create the calibration platforms.

\section{Acknowledgements}

The authors would like to acknowledge support from the National Science Foundation for the 
project Capacity and Performance of Foundations for Offshore Wind Towers, Award Number: 1041604. The authors would like to thank the Department of Aerospace Engineering at Texas A\&M University for use of their 3D printer and the faculty and staff in the Department of Civil and Environmental Engineering at Rensselaer Polytechnic Institute for their assistance.

\section{References}

Al-Defae, A. \& Knappett, J., 2014. Centrifuge modeling of the seismic performance of pilereinforced slopes. J. Geotech. and Geoenviron. Eng. ASCE 140(6): 1-13

Allmond, J.D., Hakhamaneshi, M., Wilson, D.W. \& Kutter, B.L., 2014. Advances in measuring rotation with MEMS accelerometers. Proc. of the 8th Int. Conf. on Phys. Model. in Geotech. (C. Gaudin \& D. White (eds.)). CRC Press, Perth, pp. 353-359.

Barrette, P.D., Phillips, R., Clark, J.I., Crocker, G. \& Jones, S.J., 1999. Flexural behavior of model sea ice in a centrifuge. J. of Cold Reg. Eng. ASCE 13(3): 122-138

Beemer, R.D., 2016. Experimental studies of squat gravity caissons and piles for offshore applications. Texas A\&M University.

Beemer, R.D., Aubeny, C.P. \& Biscontin, G., 2017. Centrifuge 2D gravity on a vertical rotational reference frame. Int. J. Of Phys. Modell. in Geotech. (Forthcoming).

Beemer, R.D., Murali, M., Biscontin, G. \& Aubeny, C.P., 2015. Theory on measuring orientation with MEMS accelerometers in a centrifuge. IFCEE. ASCE, San Antonio.

Bennett, V., Abdoun, T., Shantz, T., Jang, D. \& Thevanayagam, S., 2009. Design and characterization of a compact array of MEMS accelerometers for geotechnical instrumentation. Smart Struct. and Syst. Techno-Press 5(6): 663-679

Bhattacharya, S., Murali Krishna, A., Lombardi, D., Crewe, A. \& Alexander, N., 2012. Economic MEMS based 3-axis water proof accelerometer for dynamic geo-engineering applications. Soil 
Black, J.A., Baker, N. \& Ainsworth, A., 2014. Establishing a 50 g-ton geotechnical centrifuge at the University of Sheffield. 8th Int. Conf. on Phys. Model. in Geotech. Perth, Australia, pp. 181186.

Blake, A.P. \& O’Loughlin, C.D., 2015. Installation of dynamically embedded plate anchors as assessed through field tests. Can. Geotech. J. NRC Research Press 52(9): 1-13

Chow, S.H., O’Loughlin, C.D., Corti, R., Gaudin, C. \& Diambra, A., 2015. Drained cyclic capacity of plate anchors in dense sand : Experimental and theoretical observations. Geotechnique Letters. ICE 5(2): 80-85

DNV, 2007. Offshore standard DNV-OS-J101: design of offshore wind turbine structures. Hovik, Norway.

Elgamal, A., Dobry, R. \& Van Laak, P., 1991. Design, construction and operation of 100 g-ton centrifuge at RPI. Centrifuge 91. Boulder, CO, pp. 27-34.

Ellis, E., Cox, C., Yu, H., Ainsworth, A. \& Baker, N., 2006. A new geotechnical centrifuge at the University of Nottingham, UK. 6th Int. Conf. Phys. Model. Geotech. CRC Press, Hong Kong, pp. 129-133.

Hoffman, K., Varuso, R. \& Fratta, D., 2006. The use of low-cost MEMS accelerometers for the near-surface monitoring of geotechnical engineering systems. GeoCongress 2006. ASCE, Atlanta, pp. 1-6.

Lau, B.H., 2015. Cyclic behaviour of monopile foundations for offshore wind farms. University of Cambridge, Cambridgeshire.

Leung, a. M., Jones, J., Czyzewska, E., Chen, J. \& Pascal, M., 1997. Micromachined accelerometer with no proof mass. International Electron Devices Meeting. IEDM Technical Digest. 899-902

521 Madabhushi, G., 2015. Centrifuge modeling for civil engineering. CRC Press, Boca Rotan, FL. 
MEMSIC, CXL-GP series general purpose accelerometer. San Jose, CA.

MEMSIC, 2007. Ultra Low Noise, Offset Drift $\pm 1 \mathrm{~g}$ Dual Axis Accelerometer with Analog Outputs. Andover, MA.

O’Loughlin, C.D., Gaudin, C., Morton, J.P. \& White, D.J., 2014. MEMS accelerometers for measuring dynamic penetration events in geotechnical centrifuge tests. Int. J. of Phys. Modell. in Geotech. ICE 14(2): 1-9

Oppenheim, I.J., Garrett Jr., J.H. \& Ganerial, K.J., 2000. Potential MEMS applications in civil engineering. Space 2000. ASCE, Albuquerque, pp. 495-501.

Randolph, M.F., Jewell, R.J., Stone, K.J.L. \& Brown, T.A., 1991. Establishing a new centrifuge facility. Centrifuge 91. Balkema, Boulder, CO.

Saftner, D.A., Green, Russell, A., Hryciw, R.D. \& Lynch, J.P., 2008. Instrumentation for the NEESR sand aging field experiment. GeoCongress 2008. ASCE, New Orleans.

Schofield, A.N., 1969. Cambridge geotechnical centrifuge operations. Géotechnique. ICE 30(3): 227-268

Shaeffer, D.K., 2013. MEMS inertial sensors: A tutorial overview. IEEE Communications Magazine. IEEE 51(April): 100-109

Silicon Design Inc., 2013. Model 2012. Kirkland, WA.

Spangler, L. “Chip” \& Kemp, C.J., 1996. ISAAC: integrated silicon automotive accelerometer. Sensors and Actuators A: Physical. Elsevier 54(1-3): 523-529

Stark, N., Kopf, A., Hanff, H., Stegmann, S. \& Wilkens, R., 2009. Geotechnical investigations of sandy seafloors using dynamic penetrometers. OCEANS 2009, MTS/IEEE Biloxi - Marine Technology for Our Future: Global and Local Challenges.

Stratasya, 2015. Fortus: 380mc and 450 mc. Stratasy,. 
545 Stringer, M., Heron, C. \& Madabhushi, S., 2010. Experience using MEMS-based accelerometers in 546 dynamic testing. Proc. of the 7th Int. Conf. on Phys. Model. in Geotech. (C. Gaudin \& D. 547 White (eds.)). CRC Press, Perth, pp. 389-394.

548 Taboada-Urtuzuástegui, V.M. \& Dobry, R., 1998. Centrifuge modeling of earthquake-induced 549 lateral spreading in sand. J. Geotech. Geoenviron. Eng. ASCE 124(12): 1195-1206

550 Wong, H.L. \& Trifunac, M.D., 1977. Effects of cross-axis sensitivity and misalignment on the 551 response of mechanical-optical accelerographs. Bull. Seismol. Soc. Am. Seismological Society $552 \quad$ of America 67(3): 929-956.

553 


\section{Figure captions}

556 Figure 1. Example of using MEMS accelerometer to measure cyclic moment loading of a caisson.

557 Load eccentricity is 3.05 caisson diameter

558 Figure 2. Centrifuge gravity and model coordinate system from Beemer et al. (2017)

559 Figure 3. 3D printed 1-g MEMS accelerometer calibrator

560 Figure 4. Applied and measured accelerations by a MEMS Accelerometer (not to scale)

561 Figure 5. Apparent internal misalignment of the MEMS accelerometer in the x-z plane

562 Figure 6. Sketch of experiment within the centrifuge (not to scale), $\alpha$ and $\beta$ are assumed to be

563 negligible

564 Figure 7. Experiment sketch with geometry and gravity (not to scale), $\beta$ is assumed to be negligible

565 Figure 8. Sensors at Zero Degree Angle in Experiment Three Data

566 Figure 9. Measured Cross-Axis Acceleration versus Centrifuge Gravity with curve fitting, M1, M2, 567 and $\mathrm{M7}$

568 Figure 10. Results from cross-axis correction validation experiment a) Platform One b) Platform

569 Two c) Platform Three

570 
571 Table captions

572

573 Table 1. Accelerometer Technical Specifications

574 Table 2: 10 g Accelerometer Calibration Properties

575 Table 3. Experiment Targeted and Applied Reactive Centrifugal Acceleration

576 Table 4. Sensor Configuration per Experiment

577 Table 5. Measured Differential Rotation of Centrifuge Basket

578 Table 6: Results from High-g Cross-Axis Calibration of 10g Accelerometers

579 Table 7. Cross-Axis Sensitivity Validation 


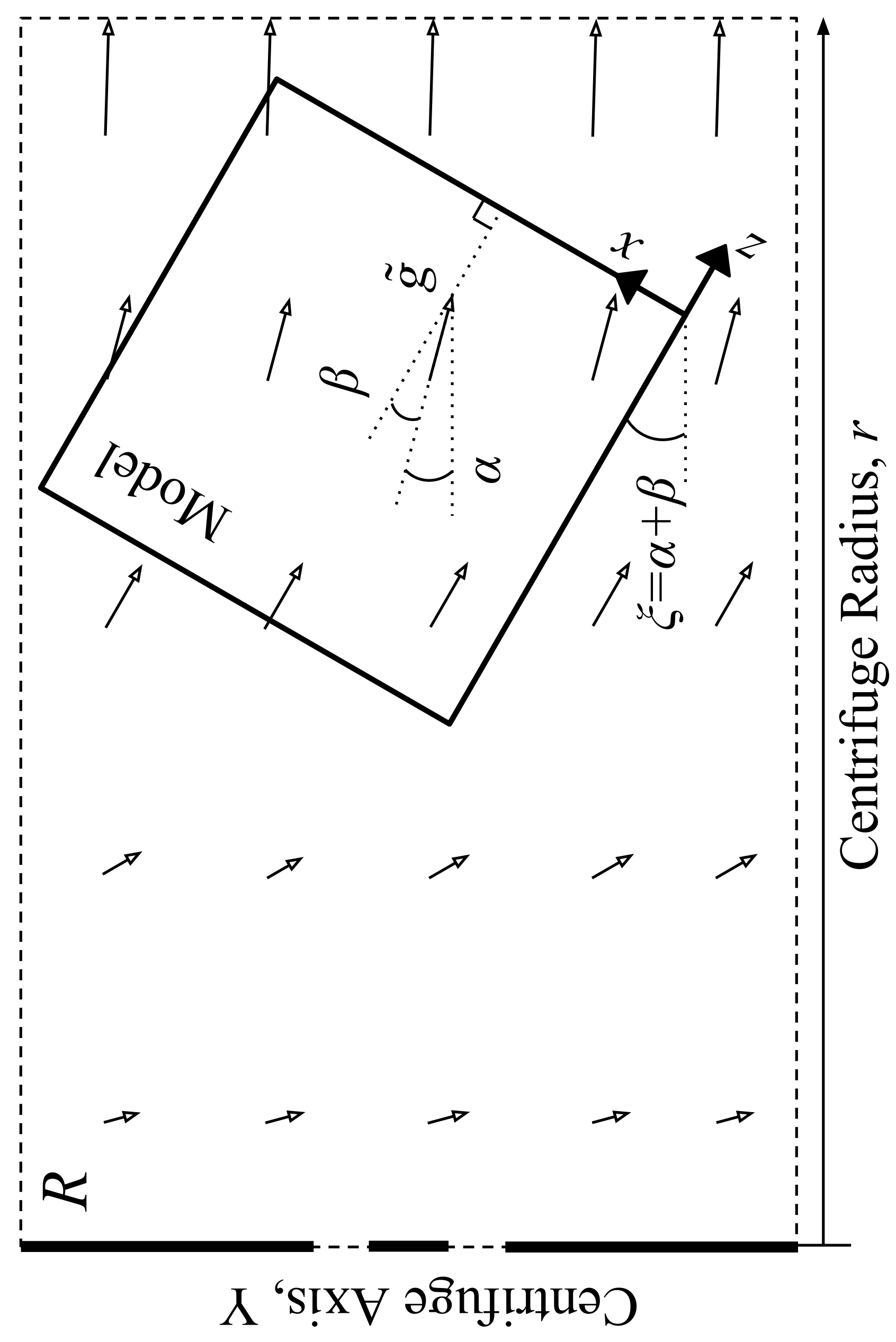

Figure02 
Figure03

Click here to download Figure Beemerfig03.JPG $\underline{\underline{ \pm}}$
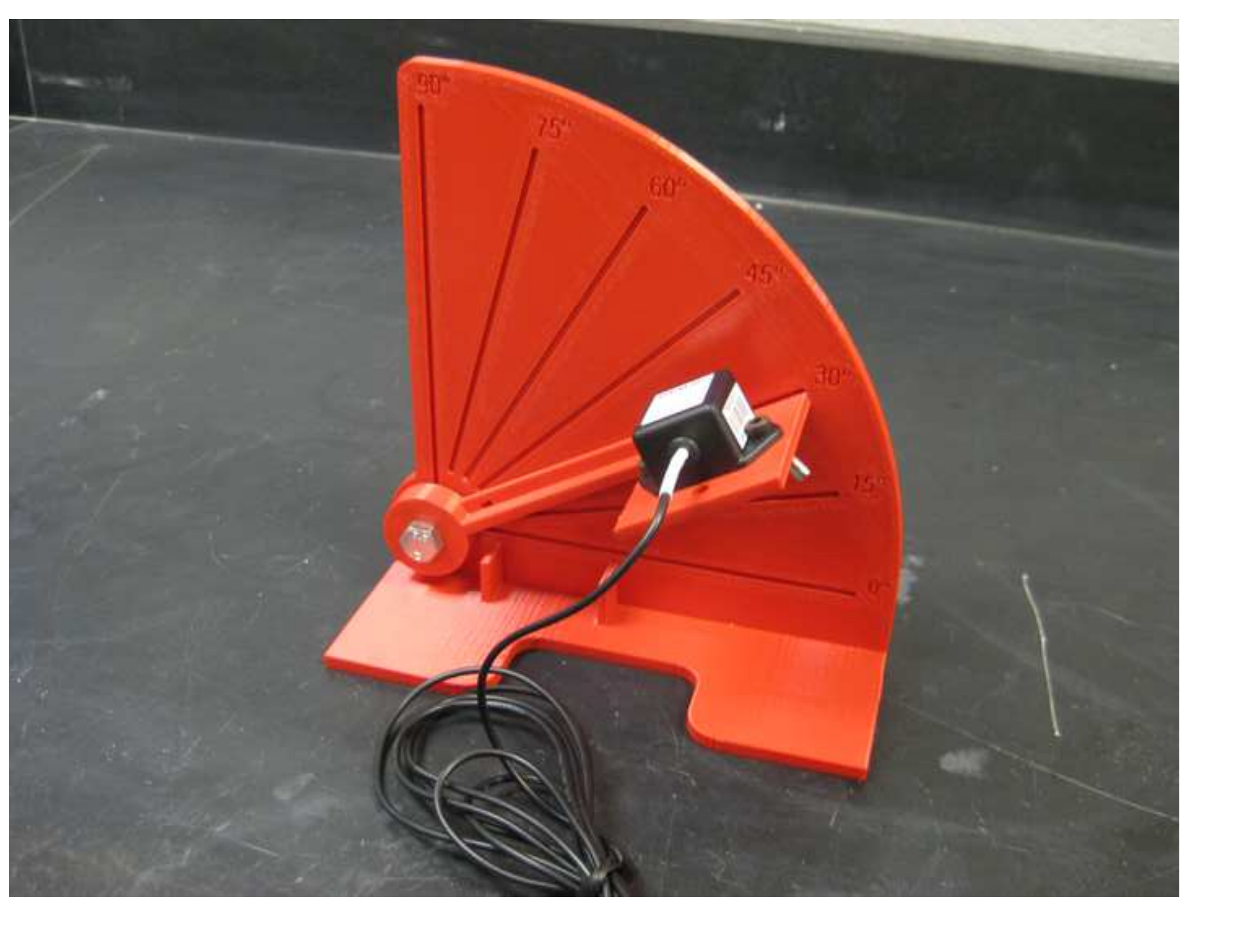

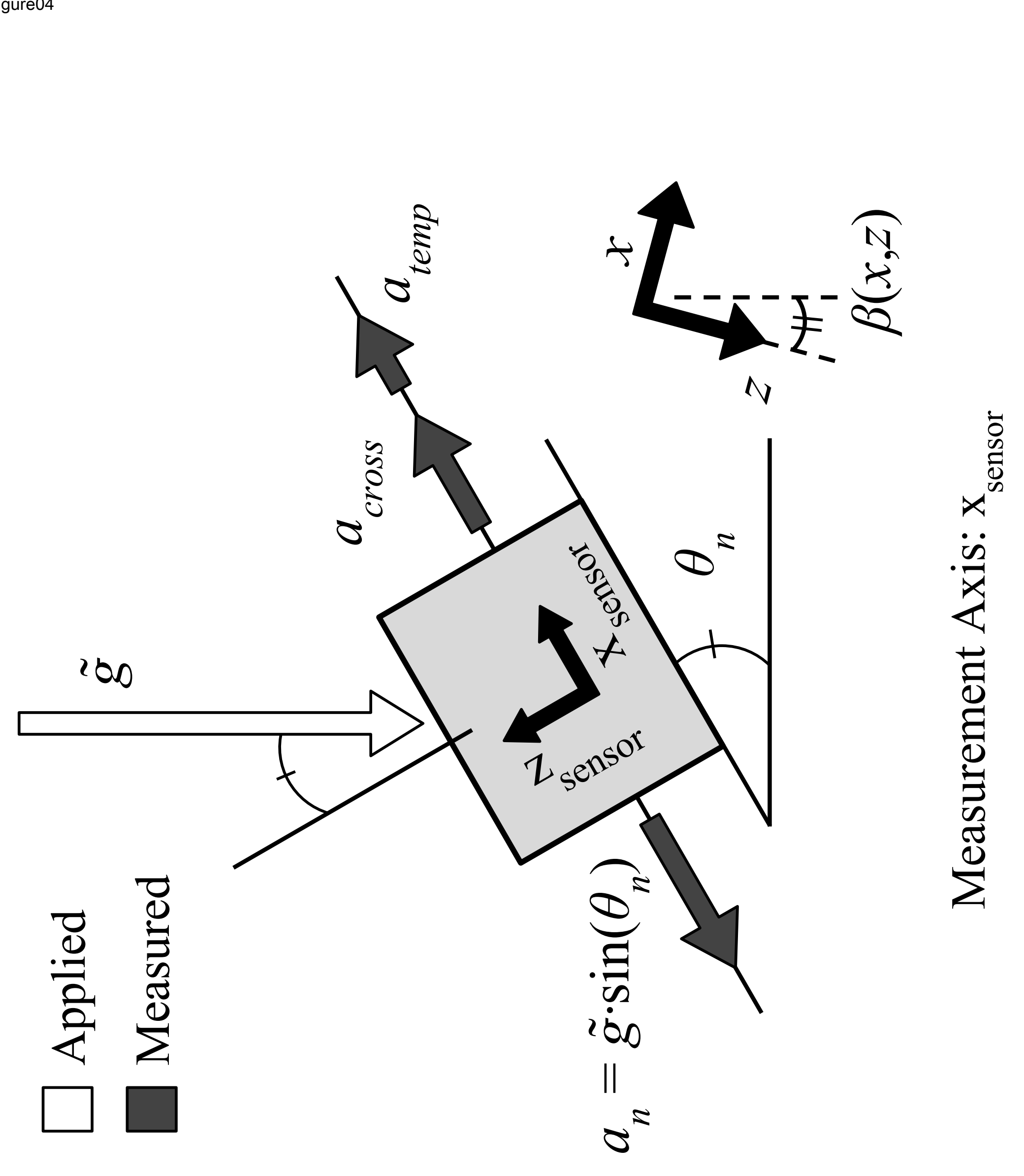

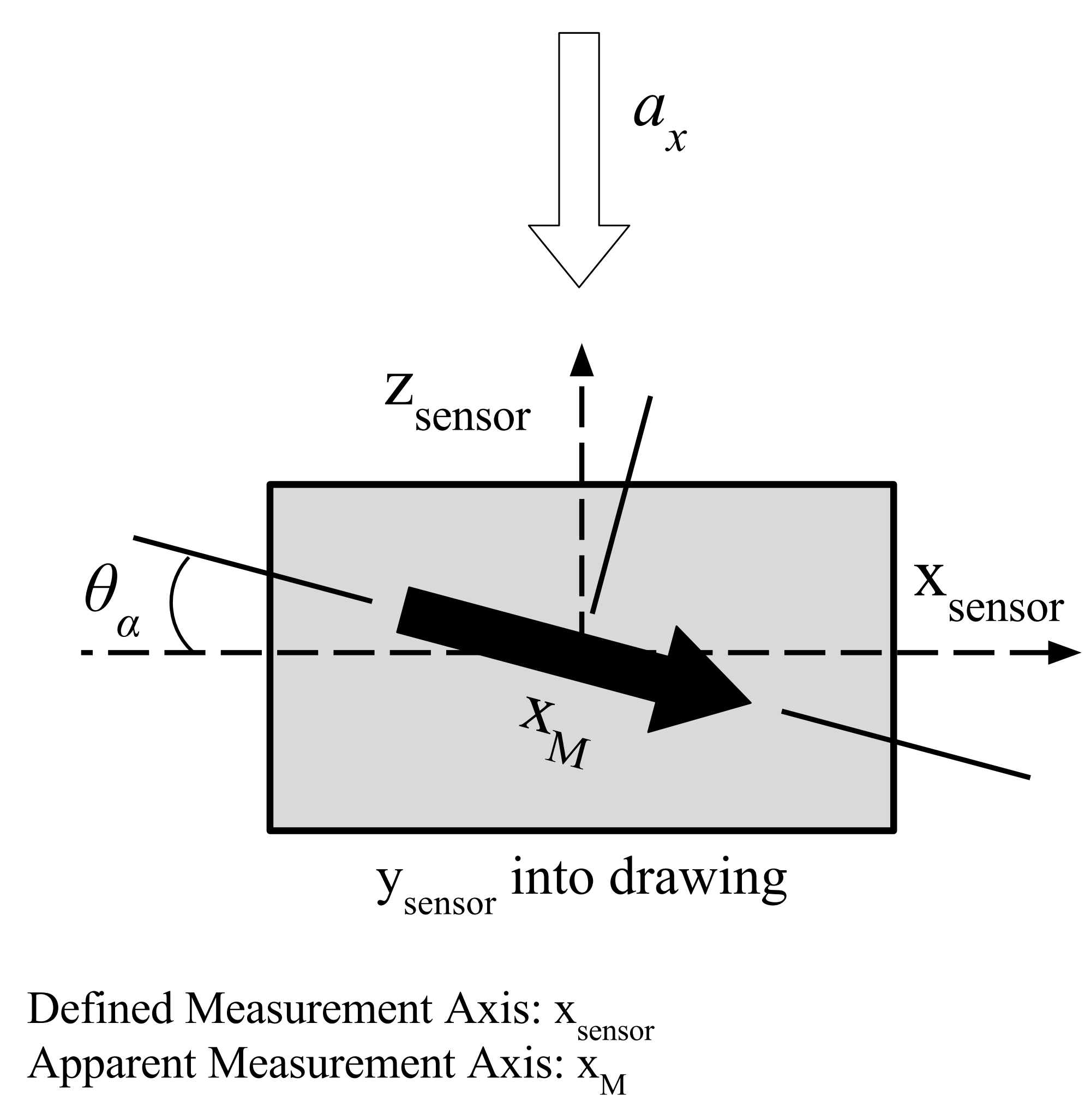

Defined Measurement Axis: $\mathrm{x}_{\mathrm{se}}$ Apparent Measurement Axis: $x_{M}$

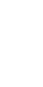

(05
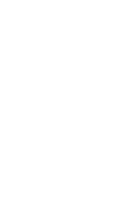

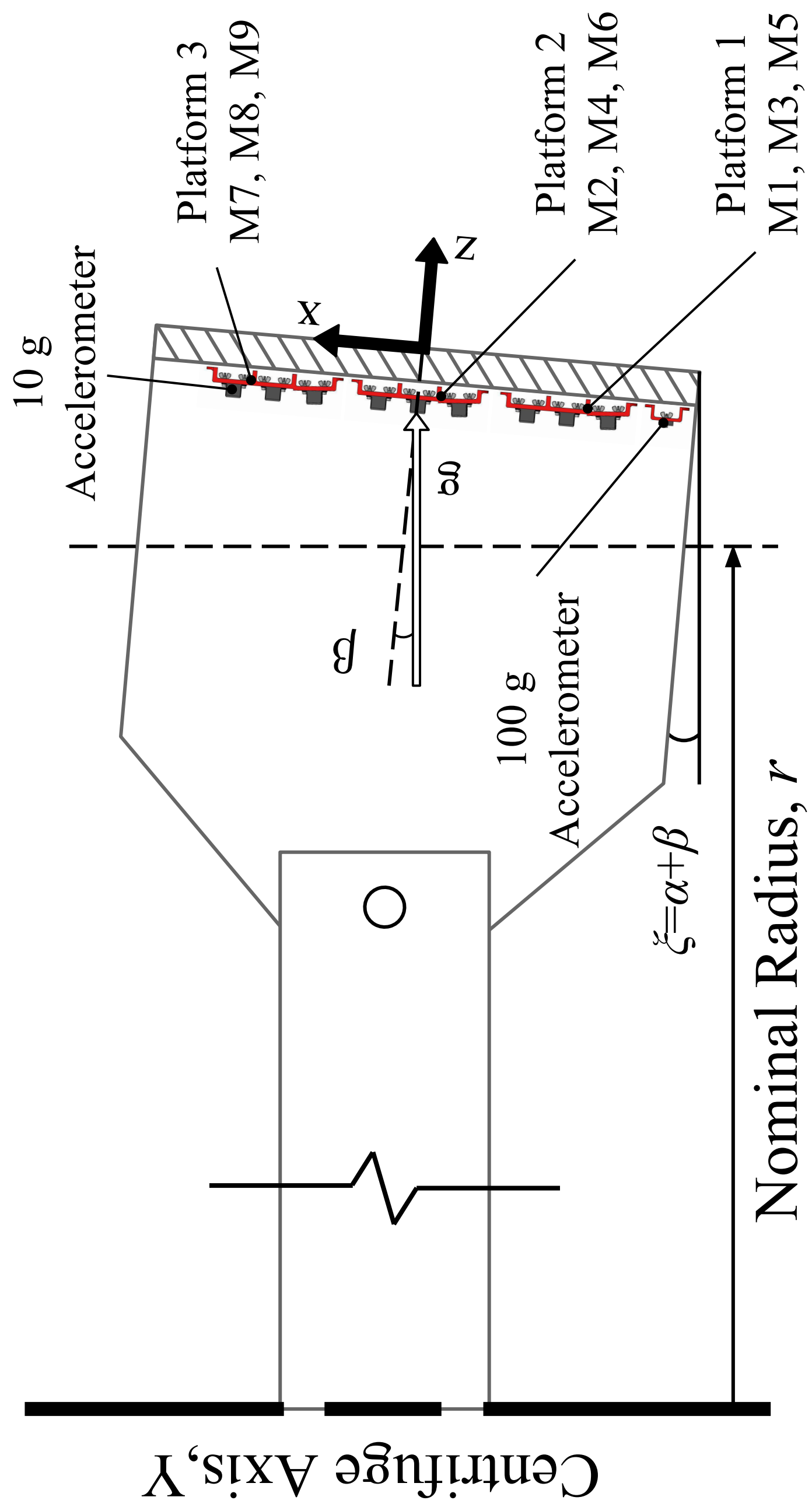


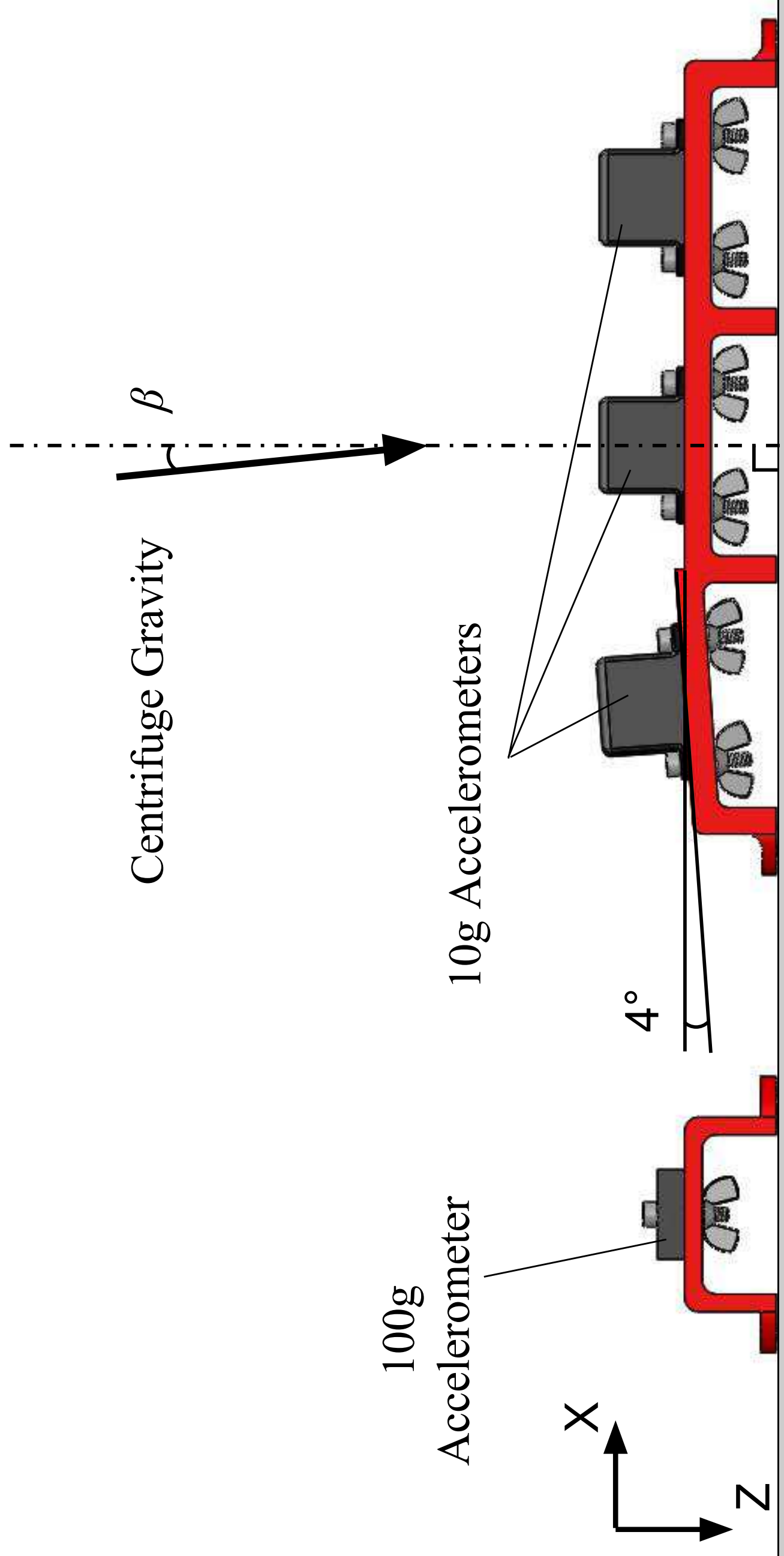




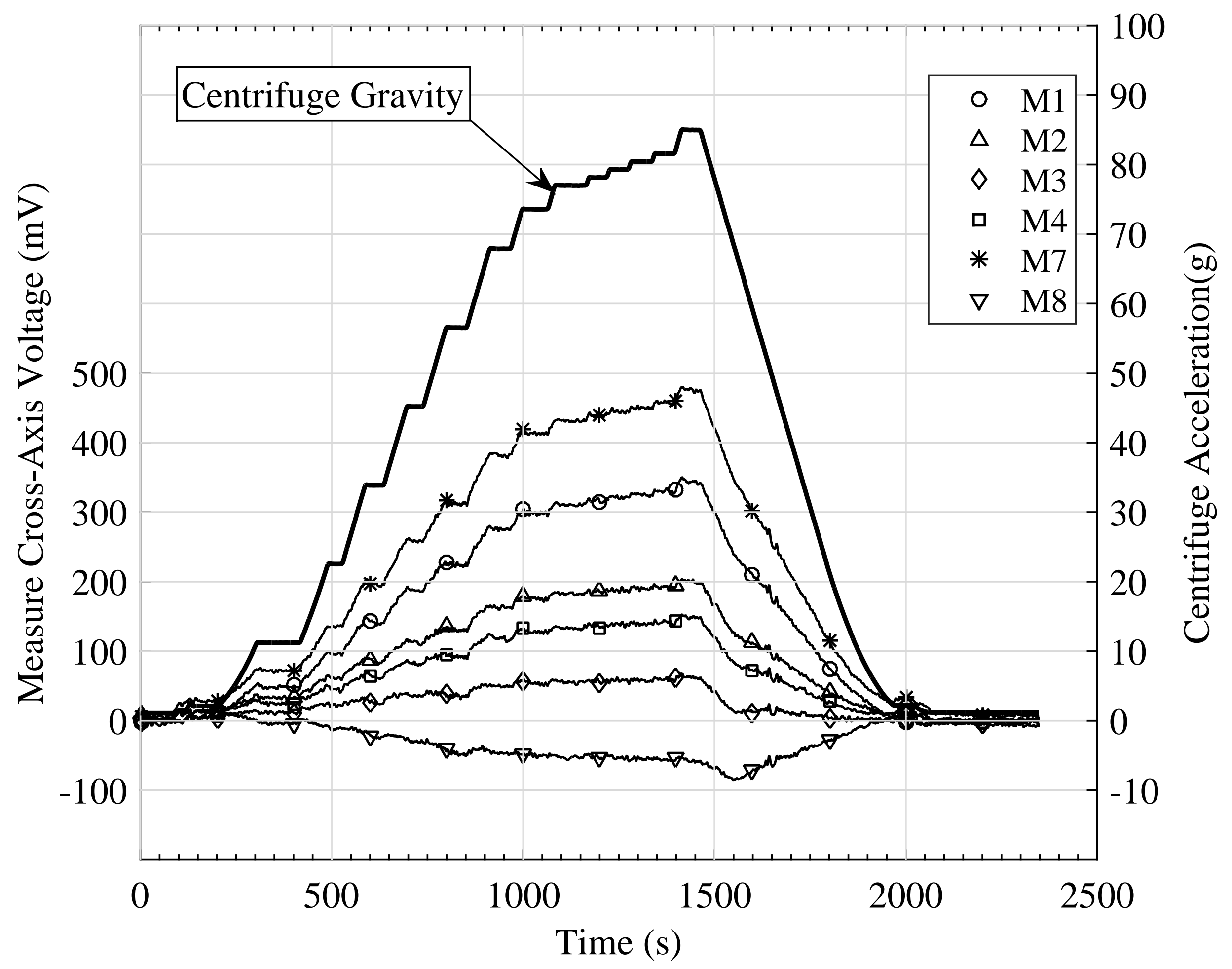




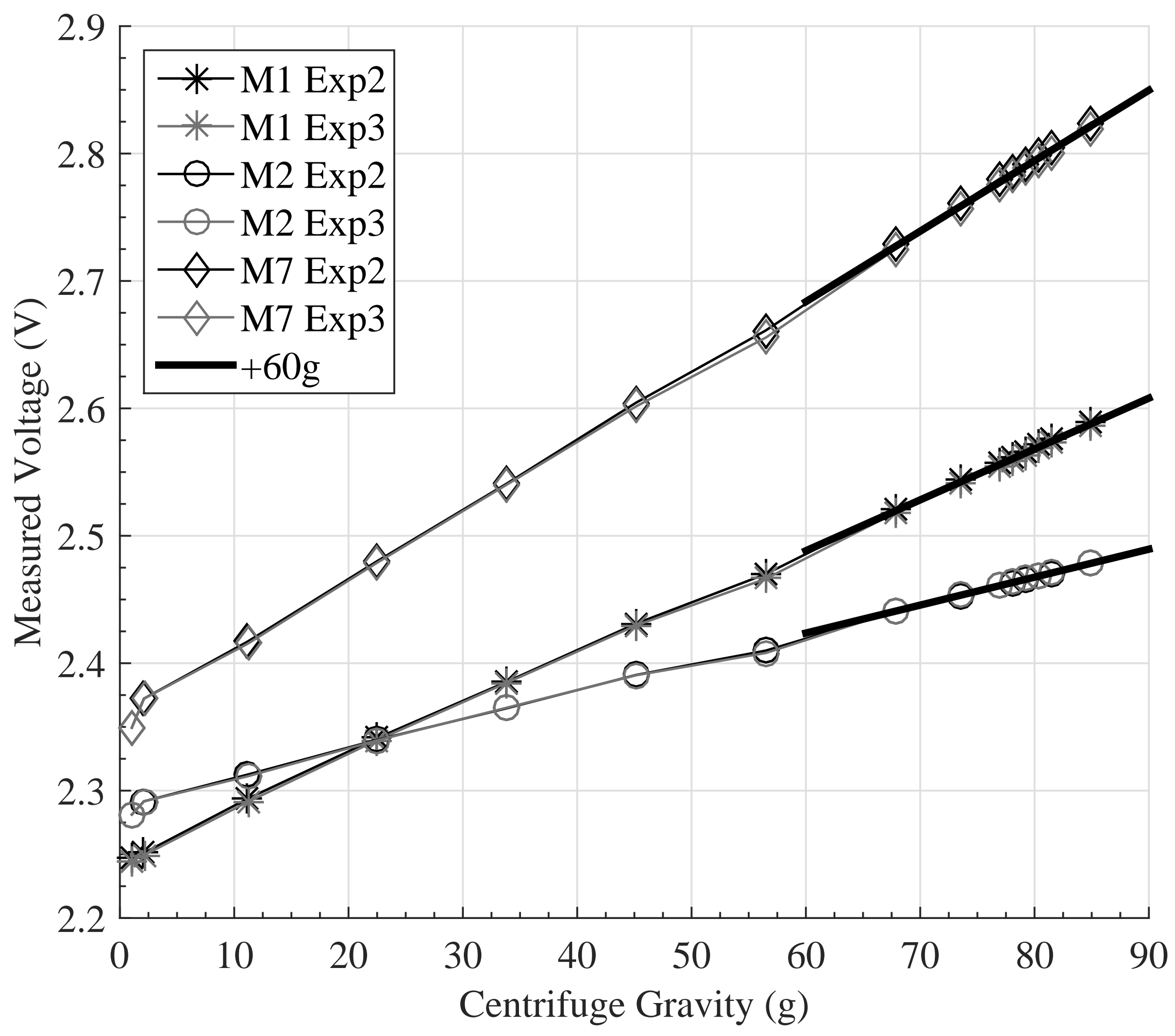


Table 1: Accelerometer Technical Specifications

\begin{tabular}{|l|c|c|}
\hline & $10 \mathrm{~g}$ Accelerometer & $100 \mathrm{~g}$ Accelerometer \\
\hline Sensitivity $(\mathrm{mV} / \mathrm{g})$ & $200 \pm 5$ & 40 \\
\hline Zero-g Voltage $(\mathrm{V})$ & $2.375 \pm 0.1$ & 2.50 (Single Ended) \\
\hline Span Output $(\mathrm{V})$ & $\pm 2.0 \pm 0.1$ & \pm 2.0 (Single Ended) \\
\hline Cross-Axis Sensitivity & $\pm 5(\%$ of Span $)$ & $2(\%) \mathrm{TYP}$ \\
\hline Alignment Error $\left(^{\circ}\right)$ & \pm 2 & - \\
\hline Noise $(\mathrm{mg} \mathrm{rms})$ & 35 & $5 \times 10^{-3} \mathrm{~g} /{ }^{\circ} \mathrm{C}$ \\
\hline Temperature Offset & $\pm 0.3 \mathrm{~g}\left(0^{\circ}-70^{\circ} \mathrm{C}\right)$ & \\
\hline
\end{tabular}


Table 2: $10 \mathrm{~g}$ Accelerometer Calibration Properties

\begin{tabular}{|c|c|c|}
\hline \multirow{2}{*}{ Sensor } & $C_{F \alpha}$ & $V_{\alpha}$ \\
\cline { 2 - 3 } & $(\mathrm{g} / \mathrm{V})$ & $(\mathrm{V})$ \\
\hline M1 & 4.982 & 2.243 \\
\hline M2 & 5.044 & 2.279 \\
\hline M3 & 5.068 & 2.321 \\
\hline M4 & 5.086 & 2.366 \\
\hline M5 & 4.993 & 2.214 \\
\hline M6 & 4.997 & 2.252 \\
\hline M7 & 4.949 & 2.344 \\
\hline M8 & 5.087 & 2.238 \\
\hline M9 & 5.029 & 2.240 \\
\hline
\end{tabular}


Table 3: Applied Cross-Axis Centrifuge Gravity

\begin{tabular}{|c|c|c|}
\hline \multirow{2}{*}{ Step } & \multicolumn{2}{|c|}{ Experiment One Centrifuge Gravity $(\mathrm{g})$} \\
\cline { 2 - 3 } & 1.00 & 1.05 \\
\hline 1 & 2.06 & 2.12 \\
\hline 2 & 22.44 & 11.19 \\
\hline 3 & 45.09 & 22.51 \\
\hline 4 & 67.77 & 33.84 \\
\hline 5 & 73.46 & 45.17 \\
\hline 6 & 76.87 & 56.51 \\
\hline 7 & 78.01 & 67.86 \\
\hline 8 & 79.16 & 73.55 \\
\hline 9 & 80.29 & 76.97 \\
\hline 10 & 81.44 & 78.10 \\
\hline 11 & 84.86 & 79.25 \\
\hline 12 & - & 80.40 \\
\hline 13 & - & 81.54 \\
\hline 14 & - & 85.95 \\
\hline 15 & & \\
\hline
\end{tabular}


Table 4: Sensor Configuration per Experiment

\begin{tabular}{|c|c|c|c|c|}
\hline \multirow{3}{*}{ Platform } & \multirow{3}{*}{ Sensor } & Experiment One & Experiment Two & Experiment Three \\
\cline { 2 - 5 } & & \multicolumn{3}{|c|}{ Orientation $\left(^{\circ}\right)$} \\
\hline \multirow{3}{*}{1} & M1 & 4 & 0 & 0 \\
\cline { 2 - 5 } & M3 & 0 & 4 & 0 \\
\cline { 2 - 5 } & M5 & 0 & 0 & 4 \\
\hline \multirow{3}{*}{2} & M2 & 4 & 0 & 0 \\
\cline { 2 - 6 } & M4 & 0 & 4 & 4 \\
\cline { 2 - 5 } & M6 & 0 & 0 & 0 \\
\hline \multirow{3}{*}{3} & M7 & 4 & 0 & 0 \\
\cline { 2 - 6 } & M8 & 0 & 4 & 4 \\
\cline { 2 - 6 } & M9 & 0 & 0 & 0 \\
\hline
\end{tabular}


Table 5: Measured Differential Rotation of Centrifuge Basket

\begin{tabular}{|c|c|c|c|c|c|}
\hline \multirow{2}{*}{$\Delta \operatorname{Exp}$} & \multirow{2}{*}{ Sensor } & Exp One & Exp Two & Exp Three & \multirow{2}{*}{$\Delta \theta_{\alpha}\left({ }^{\circ}\right)$} \\
\hline & & \multicolumn{3}{|c|}{ Misalignment, $\theta_{\alpha},\left(^{\circ}\right)$} & \\
\hline \multirow{3}{*}{$1-2$} & M3 & 0.43 & - & 0.21 & 0.23 \\
\hline & M4 & 0.67 & - & 0.50 & 0.17 \\
\hline & M8 & 0.075 & - & -0.18 & 0.25 \\
\hline \multicolumn{5}{|c|}{ Mean } & 0.22 \\
\hline \multirow{3}{*}{$1-3$} & M5 & 1.64 & 1.41 & - & 0.24 \\
\hline & M6 & 1.79 & 1.56 & - & 0.23 \\
\hline & M9 & 1.08 & 0.86 & - & 0.22 \\
\hline \multicolumn{5}{|c|}{ Mean } & 0.22 \\
\hline \multirow{3}{*}{$2-3$} & M1 & - & 1.17 & 1.16 & 0.02 \\
\hline & M2 & - & 0.64 & 0.64 & 0.00 \\
\hline & M7 & - & 1.62 & 1.60 & 0.02 \\
\hline \multicolumn{5}{|c|}{ Mean: } & 0.01 \\
\hline
\end{tabular}


Table 6: Results from High-g Cross-Axis Calibration of 10g Accelerometers

\begin{tabular}{|c|c|c|c|c|c|}
\hline \multirow{2}{*}{ Sensor } & $C_{x}$ & $\mathrm{R}^{2}$ & $\theta_{\alpha}$ & $C_{F}$ & $V_{0}$ \\
\cline { 2 - 6 } & $(\mathrm{mV} / \mathrm{g})$ & - & $\left(^{\circ}\right)$ & $(\mathrm{g} / \mathrm{V})$ & $(\mathrm{V})$ \\
\hline M1 & 3.99 & 0.999 & 1.16 & 5.086 & 2.247 \\
\hline M2 & 2.19 & 0.999 & 0.64 & 5.101 & 2.281 \\
\hline M3 & 0.72 & 0.991 & 0.21 & 5.086 & 2.321 \\
\hline M4 & 1.62 & 0.999 & 0.48 & 5.129 & 2.367 \\
\hline M5 & 4.81 & 0.999 & 1.41 & 5.119 & 2.219 \\
\hline M6 & 5.30 & 0.999 & 1.56 & 5.137 & 2.257 \\
\hline M7 & 5.56 & 0.999 & 1.61 & 5.093 & 2.349 \\
\hline M8 & -0.56 & 0.973 & -0.16 & 5.073 & 2.237 \\
\hline M9 & 2.93 & 0.999 & 0.86 & 5.105 & 2.243 \\
\hline
\end{tabular}


Table 7: Cross-Axis Sensitivity Validation

\begin{tabular}{|c|c|c|c|c|}
\hline \multirow{3}{*}{ 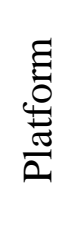 } & \multirow{3}{*}{ 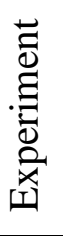 } & \multirow{3}{*}{$\begin{array}{l}\dot{0} \\
\mathscr{0} \\
\ddot{D}\end{array}$} & \multicolumn{2}{|c|}{$\begin{array}{l}\text { Average Measured Angle }\left(^{\circ}\right) \\
\text { Note: Platform Angle is } 4^{\circ}\end{array}$} \\
\hline & & & \multicolumn{2}{|c|}{ Cross-Axis Correction } \\
\hline & & & $x$ & $\checkmark$ \\
\hline \multirow{3}{*}{ 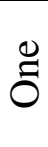 } & 1 & M1 & 2.58 & 3.81 \\
\hline & 2 & M3 & 3.58 & 3.80 \\
\hline & 3 & M5 & 2.33 & 3.82 \\
\hline \multirow{3}{*}{$\stackrel{8}{3}$} & 1 & M2 & 3.32 & 4.01 \\
\hline & 2 & M4 & 3.53 & 4.04 \\
\hline & 3 & M6 & 2.40 & 4.04 \\
\hline \multirow{3}{*}{$\stackrel{\circlearrowright}{\circlearrowright}$} & 1 & M7 & 2.28 & 3.98 \\
\hline & 2 & M8 & 4.12 & 3.94 \\
\hline & 3 & M9 & 3.06 & 3.97 \\
\hline
\end{tabular}

